\title{
Off-pump left ventricular assist device implantation via bilateral mini-thoracotomy in cardiac reoperations: the extrapericardial subxiphoid route
}

\author{
Matteo Attisani ${ }^{1 \#}$, Marco Pocar ${ }^{1,2 \#}$, Vittoria Lodo $^{1}$, Cristina Barbero ${ }^{1}$, Giovanni Marchetto ${ }^{1}$, \\ Paolo Centofanti ${ }^{1}$, Mauro Rinaldi ${ }^{1}$, Massimo Boffini ${ }^{1}$ \\ ${ }^{1}$ Department of Surgical Sciences, University of Turin, Division of Cardiac Surgery, "Città della Salute e della Scienza", Turin, Italy; ${ }^{2}$ Department of \\ Clinical Sciences and Community Health, University of Milan, Milan, Italy \\ \#These authors contributed equally to this work. \\ Correspondence to: Dr. Marco Pocar, MD, PhD. Department of Surgical Sciences, University of Turin, Division of Cardiac Surgery, "Città della Salute \\ e della Scienza”, Corso Dogliotti 14, 10126 Turin, Italy. Email: marco.pocar@unito.it.
}

Submitted Oct 29, 2020. Accepted for publication Mar 02, 2021.

doi: 10.21037/acs-2020-cfmcs-25

View this article at: http://dx.doi.org/10.21037/acs-2020-cfmcs-25

\section{Introduction}

We describe an almost entirely extrapericardial technique to implant a left ventricular assist device (LVAD) off-pump through a limited bilateral thoracotomy in reoperative cardiac surgery and prior sternotomy.

\section{Clinical vignette}

A forty-six-year-old man with ischemic cardiomyopathy, pulmonary hypertension and prior coronary artery bypass grafting underwent LVAD implantation via bilateral minithoracotomy and an extrapericardial off-pump approach. The ejection fraction was $15 \%$ and the indication was bridge to transplant candidacy.

\section{Surgical technique}

\section{Preparation}

Cardiac catheterization, echocardiography and computed tomography serve to assess biventricular and valvular function, exclude left ventricular thrombi and locate the inflow and, most importantly, the outflow anastomotic site on the ascending aorta, while identifying atheroma, calcifications and patent bypass grafts. Cutdown of the femoral vessels is prophylactically undertaken.

\section{Exposition}

An 8 to $10 \mathrm{~cm}$ left thoracotomy in the fifth (or sixth) intercostal space is performed to expose the pericardium with minimal dissection of apical adhesions and identify the left anterior descending coronary artery. Subsequently, a 5 to $6 \mathrm{~cm}$ right anterior mini-thoracotomy is performed in the second intercostal space with limited pericardial incision and dissection of adhesions to expose the ascending aorta, locate the outflow graft anastomotic site and identify bypass grafts.

\section{Operation}

Before heparinization, the inflow site ring is sewn to the left ventricle with a running 4-0 polypropylene suture, $2 \mathrm{~cm}$ from the interventricular groove. Three epicardial 2-0 polyester pledgetted mattress sutures are placed at $120^{\circ}$ for countertraction during insertion of the device.

Two-step driveline tunnelling is made to the subxiphoid region and to either of the lower abdominal quadrants, taking care to maintain a submuscular course to reduce the risk of infection. Likewise, the outflow graft is tunnelled from the left thoracotomy to the subxiphoid incision, exiting in the rectus abdominis sheath beneath the muscle belly and anterior to the visceral aponeurosis. The patient is subsequently tilted to the right and into a steep Trendelenburg position, and heparin administered 
(150 UI/kg; target activated clotting time, 250 seconds). The ventricular apex is cored, and the pump, primed with $5 \%$ dextrose and with the outflow clamped, is inserted during a one-heartbeat interval.

The distal end of the outflow graft is secured to a Foley catheter, which is inflated and used to drive the unsupported portion of the Dacron graft from the subxiphoid access to the right pleura. A side-biting clamp is placed as distally as possible on the right aspect of the ascending aorta avoiding, whenever possible, the proximal course of bypass grafts, particularly to the right coronary artery. The end-to-side anastomosis is completed with 4-0 polypropylene running suture, with the patient tilted to the left for deairing before starting LVAD support. Finally, a polytetrafluoroethylene (PTFE) membrane is sewn around the device to protect the lung and chest wall from erosion and facilitate future reoperations.

\section{Comments}

Less invasive LVAD implants are steadily increasing, supported by newer-generation centrifugal continuous-flow pumps, with the rationale of minimizing surgical trauma, blood loss and, possibly, right ventricular dysfunction (1-4).

\section{Clinical results}

Duration of mechanical ventilation was twenty hours, and the patient was discharged from the intensive care unit on postoperative day two. We have employed this technique in five patients with ischemic cardiomyopathy and prior coronary bypass grafting, with or without associated mitral or ventricular restoration surgery. Indications for LVAD were bridge-to-candidacy in four cases and destination therapy in one. All patients received a HeartWare device (HVAD, Medtronic, USA). This approach was not feasible in three additional cases because of native/prosthetic aortic regurgitation necessitating valve replacement. Operative mortality was zero and no deaths or major adverse events occurred at six-months follow-up. One patient died of hemorrhagic stroke, twelve months after implant. The first patient was successfully transplanted after sixty-five months on LVAD support.

\section{Advantages}

A sternal sparing approach is appealing in reoperative cardiac surgery and has proved feasible and safe in patients requiring LVAD (4). Benefits are further amplified by implanting the LVAD off-pump, with reduced mechanical ventilation times, lower inotropic support requirement and right ventricular dysfunction, shorter intensive care and hospital stay and better early survival (5). When considering less invasive LVAD implantation, a previous full sternotomy can be viewed as a relative contraindication due to technical constraints. However, the benefits of sternal sparing are greatest in redo patients. Limited dissection of adhesions reduces bleeding and transfusion requirements, and the consequent hazards of allosensitization in transplant candidates (1). Long-standing heart failure also predisposes to pulmonary hypertension and ventricular distension. Hence, the avoidance of further pericardial and right heart manipulation might possibly aid right ventricular function (5).

In contrast to other reports focused on reoperations, the routing of the outflow graft is truly extrapericardial. Adopting the two-step tunneling approach to the subxiphoid region and right pleura, mediastinal structures are left almost untouched, except for anastomotic sites. Alternatively, this goal may be achieved by anastomosing the outflow graft to the descending aorta or left subclavian artery, but we have no experience and favor antegrade flow in the proximal aorta. Conversely, others have described tunneling of the outflow graft behind the sternum, crossing the left ventricle and right ventricular outflow tract (2). We believe this is cumbersome and hazardous with a nonvented heart, as in off-pump procedures. Sternal-sparing LVAD implantation with the aid of cardiopulmonary bypass, including redo cases, have also been reported (4). Decompression may be achieved with adenosine and temporarily induced hypotension. Furthermore, we believe that transient asystole, or rapid ventricular pacing, might enhance air-locking during pump fixation. Pump stasis may be prevented with retrograde flushing by performing the distal anastomosis first, but we found fixation of the HVAD to the outflow graft cumbersome through a relatively small thoracotomy. We also reassembled all rings of the outflow strain relief, maintaining the graft's original configuration. This, coupled with a longer graft and a smoother curvature, has resulted in no kinking or twisting. In addition, the reinforced outflow graft remains more caudal and is less prone to iatrogenic damage during transplantation. Finally, extrapericardial subxiphoid routing not only allows a near "no-touch technique" respective to mediastinal structures, it also determines a more caudal location of the graft itself, rendering future transplantation less tedious and hazardous. 


\section{Caveats}

We are aware that further experience is necessary to validate this approach.

\section{Acknowledgments}

Funding: None.

\section{Footnote}

Conflicts of Interest: The authors have no conflicts of interest to declare.

Open Access Statement: This is an Open Access article distributed in accordance with the Creative Commons Attribution-NonCommercial-NoDerivs 4.0 International License (CC BY-NC-ND 4.0), which permits the noncommercial replication and distribution of the article with the strict proviso that no changes or edits are made and the original work is properly cited (including links to both the formal publication through the relevant DOI and the license). See: https://creativecommons.org/licenses/by-nc-nd/4.0/.

\section{References}

1. Cheung A, Lamarche Y, Kaan A, et al. Off-pump implantation of the HeartWare HVAD left ventricular assist device through minimally invasive incisions. Ann Thorac Surg 2011;91:1294-6.

2. Strueber M, Meyer AL, Feussner M, et al. A minimally invasive off-pump implantation technique for continuousflow left ventricular assist devices: early experience. J Heart Lung Transplant 2014;33:851-6.

3. Schechter MA, Patel CB, Blue LJ, et al. Improved early survival with a nonsternotomy approach for continuousflow left ventricular assist device replacement. Ann Thorac Surg 2015;99:561-6.

4. Gosev I, Wood K, Ayers B, et al. Implantation of a fully magnetically levitated left ventricular assist device using a sternal-sparing surgical technique. J Heart Lung Transplant 2020;39:37-44.

5. Mohite PN, Sabashnikov A, Raj B, et al. Minimally invasive left ventricular assist device implantation: a comparative study. Artif Organs 2018;42:1125-31.

Cite this article as: Attisani M, Pocar M, Lodo V, Barbero C, Marchetto G, Centofanti P, Rinaldi M, Boffini M. Off-pump left ventricular assist device implantation via bilateral minithoracotomy in cardiac reoperations: the extrapericardial subxiphoid route. Ann Cardiothorac Surg 2021;10(2):298-300. doi:10.21037/acs-2020-cfmcs-25 